\title{
Generación de ideas en el contexto organizacional: comprensiones a partir del estudio de una empresa del sector gráfico de Medellín*
}

Idea Generation in the Organizational Context: Understandings from a Study of a Company of the Graphic Industry in Medellin Geração de ideias no contexło organizacional: compreensões a partir do estudo de uma empresa do setor gráfico de Medellín

\author{
Manuela Escobar Sierra ${ }^{\star \star}$ \\ Luz Dinora Vera Acevedo ${ }^{* * *}$ \\ Alexander Alberto Correa Espinal ${ }^{\mid * \star \star}$
}

Fecha de recibido: 25 de julio de 2013

Fecha de aprobado: 21 de diciembre de 2013

Doi: dx.doi.org/10.12804/rev.univ.empresa.26.2014.08

Este artículo es producto de un trabajo de investigación realizado para optar al título de Magíster en Ingeniería Administrativa, en la Facultad de Minas de la Universidad Nacional de Colombia (sede Medellín).

** Magíster en Ingeniería Administrativa de la Universidad Nacional de Colombia, especialista en Gerencia de la Producción y el Servicio de la Escuela de Ingeniería de Antioquia (EIA), e integrante del grupo de investigación GEYMA de la Facultad de Minas de la Universidad Nacional de Colombia. Correo electrónico: mescobars@unal.edu.co

*** Doctora en Administración y magíster en Administración Integral del Ambiente. Profesora asistente del Departamento de Ingeniería de la Organización de la Facultad de Minas de la Universidad Nacional de Colombia. Directora del grupo de investigación GEYMA de la Facultad de Minas de esa universidad. Correo electrónico: 1dveraa@unal.edu.co

***** Doctor en Estadística e Investigación Operativa y magíster en Ingeniería Industrial de la Universidad de Los Andes. Profesor Asociado del Departamento de Ingeniería de la Organización de la Facultad de Minas de la Universidad Nacional de Colombia. Director del grupo de investigación GIMGO de la Facultad de Minas. Correo electrónico: alcorrea@unal.edu.co 
Para citar este artículo: Escobar S., M., Vera A., L. D., \& Correa E., A. A. (2014). Generación de ideas en el contexto organizacional: comprensiones a partir del estudio de una empresa del sector gráfico de Medellín. Universidad \& Empresa, 16(26), 195-213. doi: dx.doi.org/10.12804/rev.univ.empresa.26.2014.08

\section{RESUMEN}

En este artículo se analiza, a partir del estudio de una empresa del sector gráfico de Medellín (Colombia), el proceso de generación de ideas en el contexto organizacional y la caracterización, según el estilo cognitivo y las técnicas creativas, de la calidad de esas ideas. En el texto 1) se revisa la literatura sobre innovación, gestión del conocimiento y los estilos cognitivos, 2) se presenta la estrategia de investigación, que corresponde con el estudio de caso, 3) se explicita la recolección de datos realizada (que utiliza un juego gerencial compuesto por técnicas creativas, estilos cognitivos y criterios de calidad) y se muestra el análisis de los datos (que hace uso del diseño de experimentos), constatando que el juego gerencial permite focalizar las capacidades relacionadas con la gestión del conocimiento y la innovación y 4) se evidencia la relación existente entre la interacción del estilo cognitivo y las técnicas creativas, así como su impacto en la calidad de las ideas generadas.

Palabras clave: Diseño de experimentos, estilos cognitivos, generación de ideas, innovación, técnicas creativas.

\section{ABSTRACT}

Based on the study of a company of the graphic industry in Medellin (Colombia), this article analyzes the process of idea generation in an organizational context and the characterization of the quality of these ideas, according to the cognitive style and creative techniques. In the text 1) a review of literature is made of writings on innovation, knowledge management and cognitive styles, 2) the research strategy, which corresponds to the case study, is presented, 3) data collection is clearly set forth (using a management game composed of creative techniques, cognitive styles and quality criteria) and the data analysis is shown (using design of experiments), noting that the management game allows to focus the capabilities related to knowledge management and innovation, and, 4) the relationship between the interaction of cognitive style and creative techniques and their impact on the quality of generated ideas is evinced.

Keywords: Cognitive styles, Creative techniques, Design of experiments, Idea generation, Innovation.

\section{RESUMO}

Neste artigo analisa-se, a partir do estudo de uma empresa do setor gráfico de Medellín (Colômbia), o processo de geração de idéias no contexto organizacional e a caracterização, segundo o estilo cognitivo e as técnicas criativas, da qualidade dessas idéias. No texto: 1) revisa-se a literatura sobre inovação, gestão do conhecimento e os estilos cognitivos; 2) apresenta-se a estratégia de pesquisa, que corresponde com o estado de caso; 3) explicitase a recolecção de dados realizada (que utiliza um jogo gerencial composto por técnicas 
criativas, estilos cognitivos e critérios de qualidade) e se mostra a análise dos dados (que faz uso do desenho de experimentos), constatando que o jogo gerencial permite focalizar as capacidades relacionadas com a gestão do conhecimento e a inovação; e 4) evidencia-se a relação existente entre a interação do estilo cognitivo e as técnicas criativas, assim como seu impacto na qualidade das idéias geradas.

Palavras-chave: Desenho de experimentos, estilos cognitivos, geração de idéias, inovação, técnicas criativas.

\section{INTRODUCCIÓN}

La economía global ha evolucionado en las últimas décadas. Durante la década de 1980 los ejecutivos eran reconocidos por su capacidad de reestructuración; luego, durante los 90, se reconoció la habilidad para identificar y cultivar las competencias de base de la compañía (Prahalad \& Hamel, 1990), en que se vivió un proceso evolutivo que hoy por hoy se traduce en la búsqueda constante de competencias como la innovación (Alles, 2005) y la estrategia para lograr la permanencia y rentabilidad a nivel organizacional y nacional (Gomes, 2009). Sobre innovación se encuentran diversas posturas. Algunas de ellas la definen, por ejemplo, como: "la fuerza motriz que impulsa a las empresas hacia objetivos ambiciosos a largo plazo" (Comisión Europea, 1995, p. 4), otras la vinculan con "la introducción de un nuevo, o significativamente mejorado [bien o servicio], de un proceso, [o] de un nuevo método" (OECD, 2005, p. 56).
En el entorno actual, que se caracteriza por su inestabilidad, bajos presupuestos, una competencia creciente y altos índices de fracasos en lo que respecta a nuevos productos (Gomes, 2009), la innovación se convierte en un asunto estratégico. Partiendo de la necesidad de innovar a través de un nuevo o significativamente mejorado bien o servicio, se han desarrollado modelos en los que se plantean aproximaciones como la propuesta por Cooper (1990), quien, con el modelo stage-gate ${ }^{\circledR}$, analiza de comienzo a fin el proceso que debe completarse. Es importante resaltar aquí que ese proceso tiene su origen en la generación de ideas.

Reconociendo la incidencia de la generación de ideas en la innovación, es importante hacerse el siguiente cuestionamiento: ¿cómo se influencia la generación de las ideas, partiendo de las técnicas creativas y del ser humano como ser cognitivo y generador de las mismas? Esto con el fin de lograr la mayor cantidad posible de ideas de calidad, novedosas y variadas. 
Al considerar cómo se influencia la generación de ideas, diversos autores, como Howard, Dekoninck y Culley (2010) y Kohn, Paulus y Choi (2011), analizan el caso de la técnica creativa denominada lluvia de ideas. En esta misma línea, Mak y Shu (2008) estudian cómo el diseño biomimético utiliza los fenómenos biológicos para inspirar soluciones e, incluso, algunos otros autores como Wilson, Rosen, Nelson y Yen (2010) afirman que las analogías pueden ser utilizadas para ayudar en el proceso de generación de ideas.

Como se evidencia, esta primera corriente analizada se enfoca en la definición y estudio de técnicas creativas para generar ideas sin considerar aspectos cognitivos del ser humano. Sin embargo, los mencionados autores no son los únicos que plantean aproximaciones relacionadas con el tema. Hay otros que, en primer lugar, lo vinculan con los estilos cognitivos del ser humano, sin considerar las técnicas creativas (Dane, Baer, Pratt, \& Oldham, 2011; Liikkanen \& Perttula, 2006; Nijstad, Stroebe, \& Lodewijkx, 2002); en segundo lugar, lo relacionan con la generación de ideas con capacidad para resolver problemas; en tercer lugar, lo consideran como algo influenciado por los estilos cognitivos y, en cuarto lugar, plantean el modelo cognitivo de la búsqueda de la memoria (tabla 1).

Tabla 1. Revisión bibliográfica: enfoques relativos a la generación de ideas

\begin{tabular}{|c|c|c|}
\hline Técnicas creativas & $\begin{array}{c}\text { Técnicas creativas y estilo } \\
\text { cognitivo }\end{array}$ & Estilo cognitivo \\
\hline $\begin{array}{l}\text { - La técnica creativa: lluvia de } \\
\text { ideas (Howard et al., 2010). } \\
\text { - Diseño biomimético (fenó- } \\
\text { menos biológicos para inspi- } \\
\text { rar soluciones) (Mak \& Shu, } \\
\text { 2008). } \\
\text { - Construcción de las ideas con } \\
\text { lluvia de ideas (Kohn et al., } \\
\text { 2011). } \\
\text { - Analogías para la generación } \\
\text { de nuevas ideas (Wilson et } \\
\text { al., 2010). }\end{array}$ & $\begin{array}{l}\text { - Hasta el momento no se ha } \\
\text { encontrado un autor que inte- } \\
\text { gre ambos enfoques. }\end{array}$ & $\begin{array}{l}\text { - Generación de ideas y capa- } \\
\text { cidad de resolver problemas } \\
\text { influenciada por los estilos } \\
\text { cognitivos (Dane et al., 2011). } \\
\text { - Modelo cognitivo de la bús- } \\
\text { queda de la memoria en la ge- } \\
\text { neración de ideas (Liikkanen } \\
\text { \& Perttula, 2006). } \\
\text { - Los efectos del intercambio } \\
\text { de ideas en los procesos cog- } \\
\text { nitivos (Nijstad et al., 2002). }\end{array}$ \\
\hline
\end{tabular}

Fuente: Elaboración propia. 
Luego de encontrar y analizar estas aproximaciones la pregunta planteada continúa vigente, debido a que no es claro el método o la técnica que permita controlar o influenciar positivamente la generación de las ideas, sin dejar de lado el hecho del ser humano en cuanto tal. Ser que, entre otras dimensiones, es cognitivo. Es alguien con capacidad para resolver problemas y usar las técnicas creativas existentes. Con el objetivo de resolver este interrogante, se plantea como estrategia de investigación el estudio de caso para modelar el proceso de generación de ideas en el ambiente organizacional. Se considera como método de recolección de datos a los juegos gerenciales, las técnicas creativas, el cuestionario de la Dra. Benziger de los perfiles cognitivos (Chernigovskaya, Gavrilova, Voinov, \& Strel'nikov, 2005) y la métrica de calidad establecida por Reinig y Briggs (2013). Para el análisis de resultados se usa el diseño de experimentos (modelo factorial mixto) y, finalmente, como unidad de análisis (población estudiada) se cuenta con 250 colaboradores de una empresa del sector gráfico de la ciudad de Medellín.

En el primer numeral se revisa la literatura sobre innovación, gestión del conocimiento $\mathrm{y}$ estilos cognitivos. En la sección de meto- dología se consideran, de acuerdo con lo indicado en el párrafo anterior, el estudio de caso, el diseño de experimentos, las técnicas creativas, los juegos gerenciales, la clasificación del estilo cognitivo y los criterios de calidad de las ideas. En el tercer numeral se relacionan los resultados obtenidos. En el cuarto numeral se incorpora la interpretación y la discusión de esos resultados $\mathrm{y}$, por último, se presentan las conclusiones y recomendaciones. Todo esto se realiza con el fin de aportar a una más amplia comprensión sobre la estructuración de los equipos de trabajo y las técnicas de generación de ideas propicias, según la composición cognitiva del equipo. Lo anterior, además, permite optimizar el primer proceso de la innovación. Se pretende, finalmente, entender las dinámicas de los colaboradores, lo cual genera una mayor posibilidad de alcanzar niveles de competitividad que puedan ser replicados para otras industrias.

\section{REVISIÓN DE LA LITERATURA}

En esta sección se revisan las teorías y los autores más relevantes y cercanos al objetivo del estudio. Todos ellos vinculados de algún modo con los temas de la innovación, la gestión del conocimiento y los estilos cognitivos. 


\section{A. Innovación}

El concepto innovación comienza a vislumbrarse en los escritos de Adam Smith y David Ricardo (1817). Ellos hacían referencia a la invención de herramientas y máquinas por parte del trabajador (Formichella, 2005). Bajo esta primera aproximación surgen diversas teorías sobre la innovación, entre estas las que la relacionan estrechamente con el cambio tecnológico. Estas aproximaciones se caracterizan por la búsqueda de incentivos, la reducción de los costos, los esfuerzos de investigación y desarrollo y el capital humano (Jardón, 2011; Schumpeter, 1934). Durante el desarrollo del artículo se utiliza como referente básico el trabajo clásico de Schumpeter (1934).

Durante las décadas de 1970 y 1980 surgen otras aproximaciones teóricas. La idea que une estas aproximaciones es la concepción del desarrollo tecnológico como un proceso evolutivo, dinámico, acumulativo y sistémico (Méndez, 2002; Pérez \& Carrillo, 2000). La llamada teoría de la empresa afirma que la innovación está influenciada por el entorno general en el que la compañía opera y el sector económico en el que ella realiza su actividad, así como su estrategia competitiva, disponibilidad de recursos, tamaño, dirección y cultura empresarial (De Brentani \& Kleinschmidt, 2004). En un proceso evolutivo de la teoría se presentan nuevas aproximaciones, en donde se postula que sobre la innovación influyen los proveedores, las redes colectivas, la cooperación tecnológica, los recursos naturales, la necesidad de financiación y las estrategias de producción y marketing (Porter, 1985; Verhees \& Meulenberg, 2004). Finalmente, se encuentran posturas, como las de Johannessen, Olsen y Olaisen (1999), que definen a la teoría de la innovación sobre la base de la visión de la organización y la gestión del conocimiento.

\section{B. Gestión del conocimiento}

Son varias las teorías que se han desarrollado para abordar el tema, entre estas se encuentran los planteamientos de Nonaka y Takeuchi, (2000), cuyas principales características se relacionan con la concepción del conocimiento como una espiral, de contenido epistemológico y ontológico, en donde el punto de inicio es la persona. El objetivo, en este contexto, es poner todo el conocimiento personal a disposición de los otros, lo que es postulado y referente de esta investigación. Otros autores, como Bueno (2007), plantean que la gestión del conoci- 
miento está basada en la creación del mismo, definida esta actividad como un conjunto de métodos, técnicas, herramientas, métricas y tecnologías que permiten obtener el conocimiento en el momento oportuno, de modo fácil y rápido. Riesco (2006), por su parte, incluye el concepto de innovación en su definición de gestión del conocimiento. Finalmente, Lin y Huang (2008) reconocen que los modelos de gestión del conocimiento no siempre son exitosos debido a que no consideran aspectos cognitivos propios del ser humano.

\section{Estilo cognitivo}

Estudiado desde la psicología cognitiva, este concepto inicialmente relacionaba tres enfoques complementarios (personalidad, cognición y habilidades mentales). Luego la psicología permitió comprender los estilos con los que los individuos responden a sus distintas labores (de Zubiría Samper, Cortés, $\&$ Rodríguez, 2007). Con ello se le dio un sentido más concreto a este término, el cual supone que diferentes personas procesan la misma información de diferentes maneras, usando áreas cerebrales distintas, tal y como lo referencia Gardié (1998) y lo soporta Tennant (2006). Este último autor afirma que el estilo cognitivo se refiere a una "carac- terística individual y enfoque consistente para organizar y procesar la información" (Tennant, 2006, p. 89). Esta aproximación proporciona evidencias sobre la asimetría funcional del cerebro humano (Carrión, 1995; Sperry, 1968).

Gardner (1994), por otra parte, plantea la teoría de las inteligencias múltiples. Esta teoría se caracteriza principalmente por afirmar que las habilidades dependen de las zonas cerebrales. En línea con esta teoría, MacLean (1990) plantea el modelo del cerebro, basado en su desarrollo evolutivo, al que se denomina la teoría del cerebro triuno (o triúnico). Sugiere que el cerebro humano es en realidad tres cerebros en uno: el sistema reptiliano o Complejo-R, el sistema límbico y el neocórtex.

A su vez, autores como Riding, Glass y Douglas (1993), examinan los principales estilos cognitivos que han sido utilizados en la investigación y los clasifican en dos grandes categorías: estilo totalizador-analítico y estilo verbal-imaginativo. A partir de análisis empíricos, autores como Springer y Deutsch (1988) vinculan los estilos de pensamiento con los hemisferios cerebrales. Un enfoque muy interesante es el propuesto por Herrmann (1991), conocido como el modelo del ce- 
rebro total, que resulta de la integración de la teoría de especialización hemisférica y de la teoría del cerebro triuno. En este enfoque el procesamiento de la información lo constituyen cuatro partes o cuadrantes. Este es un enfoque tomado como referente en la investigación.

\section{METODOLOGÍA}

Como ya se ha anunciado en la introducción de este texto, para el desarrollo del presente trabajo de investigación se consideró la revisión de múltiples métodos y técnicas, a saber: estudio de caso, técnicas creativas, método para la clasificación del estilo cognitivo, diseño de juegos gerenciales, calidad de las ideas generadas y diseño de experimentos.

\section{A. Estudio de caso}

El estudio de caso es un método que inicialmente fue caracterizado por su utilidad en los procesos de investigación (Yin, 1992), posteriormente se evidenció la relevancia del método como herramienta de evaluación. Este método está compuesto de las siguientes etapas: diseñar el caso de estudio, conducirlo, analizar las evidencias y desarrollar las conclusiones, recomendaciones e implicaciones del mismo.

\section{B. Técnicas creativas}

Con base en los planteamientos de Schumpeter (1934), se hace uso de técnicas creativas que estimulan el pensamiento, la imaginación y la generación de ideas, desde una amplia variedad de actividades (Vieira, Alves, \& Duboc, 2012).

\section{Método para clasificar el estilo cognitivo del ser humano}

Partiendo del postulado propuesto por Herrmann (1991), conocido como el modelo del cerebro total, el método propuesto para la clasificación del estilo cognitivo toma como referencia el cuestionario de la Dra. Benziger. Este instrumento está compuesto por preguntas, agrupadas por segmentos o dominancias cerebrales, en donde cada persona debe responder afirmativa o negativamente. Esto ha sido trabajado en diversos estudios (Chernigovskaya et al., 2005).

\section{Método para el diseño de juegos gerenciales}

Tomando como referente el planteamiento de Nonaka y Takeuchi (2000), relacionado con la gestión del conocimiento, se selecciona el juego gerencial como método para modelar el proceso. El procedi- 
miento para diseñar el juego gerencial se basa en el trabajo de Gómez (2010). Este procedimiento consta de diez pasos que es necesario llevar a cabo. Luego de desarrollar cada uno de los pasos, se consolida un juego gerencial, en donde se hace la clasificación del estilo cognitivo y se aplican técnicas creativas como análisis morfológico, SCAMPER (i.e., sustituir, combinar, adaptar, modificar, poner en otros usos, eliminar y reformar) y analogía con imágenes y sombreros para pensar. Esto con el objetivo de generar ideas y evaluar su calidad.

\section{E. Método para medir la calidad de las ideas generadas}

Para evaluar la calidad de las ideas generadas se adopta la metodología propuesta por Reinig y Briggs (2013). En ella, a través de una escala de Likert, los expertos del proceso determinan la calificación de la idea (siendo 1 la puntuación más baja y 3 , la más alta).

\section{F. Método para el diseño de experimentos}

La metodología seleccionada para el diseño del experimento es la propuesta por Montgomery (2005). Los elementos principales de esta metodología se detallan a continuación:
Población y muestra: La población de colaboradores analizada está compuesta por 250 operarios y líderes de los procesos productivos de la compañía. De esta población se analizó una muestra representativa, compuesta por 26 líderes. Ellos fueron seleccionados bajo criterios de aleatorización (esta prueba se ejecutó el 16 de mayo de 2013).

Procesamiento de datos: El software estadístico seleccionado para realizar el proceso es Minitab ${ }^{\circledR}$ versión 16, con su procedimiento ANOVA balanceado.

Factores: Se consideró como factor de efectos fijos al estilo cognitivo dominante (factor que corresponde con la población total de estilos cognitivos descubiertos hasta ahora, por lo que es considerado como un factor de efectos fijos) y de efectos aleatorios a la técnica creativa (factor que hace parte de la población de técnicas disponibles, por lo que este es considerado un factor de efectos aleatorios).

Técnica: Se seleccionó la técnica de diseño de experimentos factoriales (modelo mixto).

Variable respuesta: Corresponde a la calidad de las ideas generadas (Reinig \& Briggs, 2013), en donde 
la calidad es definida por un experto en el proceso, quien las califica según un escala de Likert de 1 a 3. La variable respuesta es la suma de la calidad de las ideas generadas por cada persona en cada técnica.

Aleatorización: Se consideró tanto la ejecución de la prueba experimental como la aplicación del material durante la prueba, en orden aleatorio.

El modelo que describe los diseños factoriales con efectos aleatorios y fijos se presenta a continuación (Montgomery, 2005):

1. $y_{i j k}=\mu+\tau_{i}+\beta_{j}+\tau \beta_{i j}+\varepsilon_{i j k}$

En donde:

$\tau_{i}$ Es un efecto fijo (estilo cognitivo).

$\beta_{j}$ Es un efecto aleatorio (técnica creativa).

$\tau \beta_{i j}$ Es la interacción de ambos efectos que se supone aleatoria (interacción estilo cognitivo/técnica creativa).

$\varepsilon_{i j k}$ Error aleatorio.

Las hipótesis nulas para probar el modelo son:

2. $H_{0}: \tau_{i}=0$ Estadístico de prueba para probar que las medias del efecto fijo son cero.
3. $H_{0}: \sigma_{\beta}^{2}=0$ Estadístico de prueba para probar que la varianza del efecto aleatorio es cero.

4. $\mathrm{H}_{0}: \sigma_{\tau \beta}^{2}=0$ Estadístico de prueba para testear si la varianza del efecto aleatorio (interacción entre efecto fijo y aleatorio) es cero.

\section{RESULTADOS}

Durante la primera prueba de clasificación del estilo cognitivo los participantes fueron sometidos a una serie de preguntas relacionadas con sus preferencias personales. Esto con el fin de determinar su estilo cognitivo. Los resultados obtenidos fueron los siguientes: para el estilo cognitivo experimentar se presentaron dos personas; para organizar, seis; para razonar, cuatro; para sentir, siete, y para dominancias múltiples, siete también.

Con esta clasificación se evidencia que el estilo cognitivo menos frecuente en la muestra experimental se relaciona con el de experimentar. Este solo se encontró en dos personas. Es un aspecto que restringe a dos el número de réplicas con las que se realiza el experimento. Por lo tanto, la unidad experimental está compuesta por ocho personas. Cada una aporta cuatro resultados de calidad (un resultado por técnica), con lo que 
finalmente se tienen treinta y dos observaciones a analizar.

Cada persona inició el juego con la técnica relacionada con su estilo cognitivo dominante. Con esto se buscó contribuir a la aleatoriedad de la prueba. A medida que completaban las técnicas, las ideas generadas eran consignadas, para luego ser revisadas, una a una, por el equipo de expertos. Los resultados de calidad obtenidos se relacionan en la siguiente tabla:

Tabla 2. Resultado de calidad para la primera prueba experimental

\begin{tabular}{|c|c|c|c|}
\hline $\begin{array}{l}\text { Código de } \\
\text { la persona }\end{array}$ & Perfil dominante & Técnica & $\begin{array}{c}\text { Suma calidad } \\
\text { de las ideas }\end{array}$ \\
\hline 2 & Sentir & Analogía con imágenes & 9 \\
\hline 4 & Sentir & Sombreros para pensar & 6 \\
\hline 5 & Organizar & SCAMPER & 1 \\
\hline 10 & Organizar & SCAMPER & 2 \\
\hline 22 & Razonar & Análisis morfológico & 10 \\
\hline 24 & Razonar & Análisis morfológico & 8 \\
\hline 25 & Experimentar & Analogía con imágenes & 7 \\
\hline 26 & Experimentar & Analogía con imágenes & 12 \\
\hline 2 & Sentir & SCAMPER & 13 \\
\hline 4 & Sentir & Análisis morfológico & 1 \\
\hline 5 & Organizar & Sombreros para pensar & 6 \\
\hline 10 & Organizar & Análisis morfológico & 6 \\
\hline 22 & Razonar & SCAMPER & 10 \\
\hline 24 & Razonar & SCAMPER & 10 \\
\hline 25 & Experimentar & Análisis morfológico & 5 \\
\hline 26 & Experimentar & SCAMPER & 9 \\
\hline 2 & Sentir & Sombreros para pensar & 3 \\
\hline 4 & Sentir & Analogía con imágenes & 5 \\
\hline 5 & Organizar & Análisis morfológico & 1 \\
\hline 10 & Organizar & Sombreros para pensar & 5 \\
\hline 22 & Razonar & Sombreros para pensar & 7 \\
\hline 24 & Razonar & Sombreros para pensar & 1 \\
\hline 25 & Experimentar & SCAMPER & 7 \\
\hline 26 & Experimentar & Sombreros para pensar & 11 \\
\hline
\end{tabular}




\begin{tabular}{c|l|l|c}
\hline $\begin{array}{c}\text { Código de } \\
\text { la persona }\end{array}$ & Perfil dominante & Técnica & $\begin{array}{c}\text { Suma calidad } \\
\text { de las ideas }\end{array}$ \\
\hline 2 & Sentir & Análisis morfológico & 1 \\
\hline 4 & Sentir & SCAMPER & 9 \\
\hline 5 & Organizar & Analogía con imágenes & 4 \\
\hline 10 & Organizar & Analogía con imágenes & 3 \\
\hline 22 & Razonar & Analogía con imágenes & 1 \\
\hline 24 & Razonar & Analogía con imágenes & 1 \\
\hline 25 & Experimentar & Sombreros para pensar & 5 \\
\hline
\end{tabular}

Fuente: Elaboración propia.

Una vez ingresados los datos al software estadístico Minitab ${ }^{\circledR}$, el primer paso fue verificar el cumplimiento de los supuestos del modelo:

Supuesto de normalidad: Aquí se verifica si se satisface el supuesto de $N I D\left(0, \sigma^{2}\right)$ para los errores (Montgomery, 2005). Para hacerlo, existen métodos gráficos y métodos analíticos como el de Anderson Darling (Villaseñor, 2011), que, en este caso, fue el que se empleó.
Hipótesis a verificar:

\section{5. $H_{0}$ : Distribucion normal $H_{1}$ : Distribucion diferente a la normal}

Para un valor de $\alpha=0,05$, en el Gráfico 1, se presenta la distribución de probabilidad de la muestra y los resultados de los estadísticos de prueba. De allí se obtiene el valor $p=0,076 \mathrm{y}$, por tanto, valor $p>\alpha$, de donde se concluye la NID $\left(0, \sigma^{2}\right)$ para los errores.

Figura 1. Grafica de probabilidad normal y prueba de Anderson Darling para la prueba experimental.

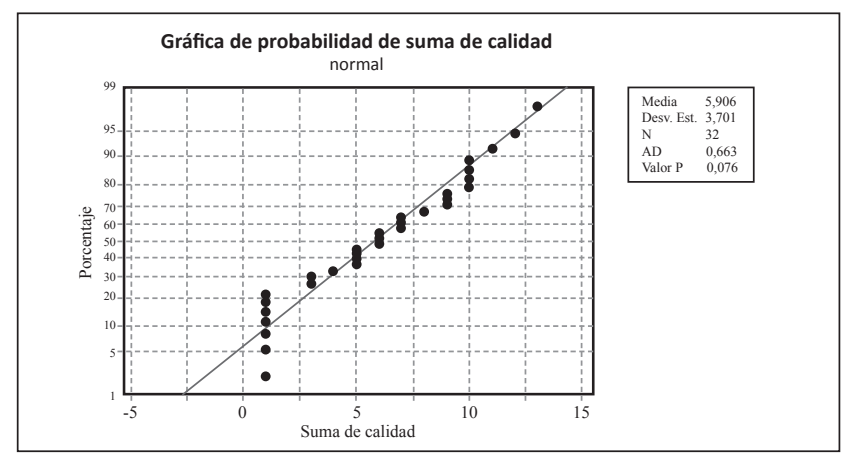

Fuente: Elaboración propia. 
Prueba de homogeneidad de varianzas: En donde se verifica que los residuales no tengan una estructura definida y no estén relacionados con ninguna otra variable. Aunque es frecuente el uso de las gráficas residuales para diagnosticar la desigualdad de la varianza, se han propuesto varias pruebas estadísticas para comprobarlo. Entre ellas, la prueba de Bartlett, que es la que se emplea en este caso (Montgomery, 2005).
Hipótesis a verificar (Melo Martínez, López Pérez, \& Melo Martínez, 2007):

6. $\mathrm{H}_{0}: \sigma_{1}^{2}=\sigma_{2}^{2}=\cdots=\sigma_{\mathrm{k}}^{2}$

$$
\mathrm{H}_{1}: \sigma_{\mathrm{i}}^{2} \neq \sigma_{\mathrm{i}^{\prime}}{ }^{2}
$$

Para un valor de $\alpha=0,05$, (ver Gráfico 2), se obtiene el valor $p=0,917$ $($ valor $p>\alpha)$.

Figura 2. Prueba de igualdad de varianzas y prueba de Bartlett para la prueba experimental

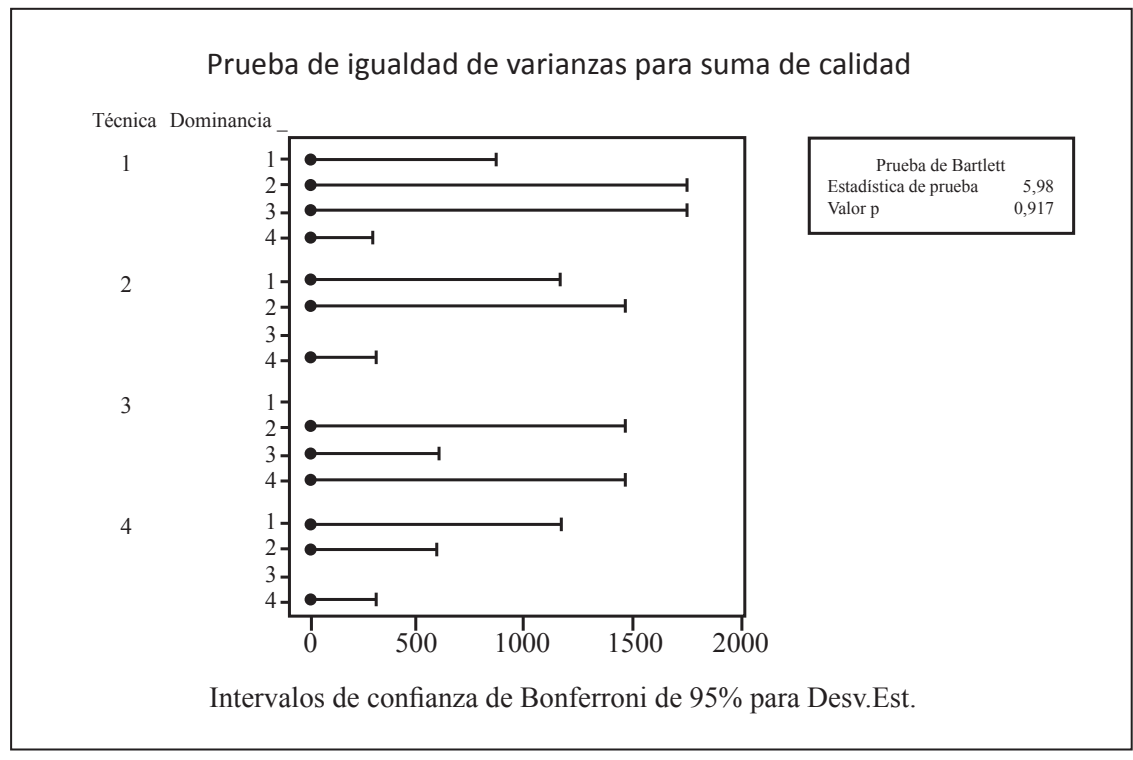

Fuente: Elaboración propia.

De allí se puede afirmar, con un nivel de confianza del $95 \%$, que las varianzas son iguales. Luego de verificados los supuestos, se procede con el cálculo del ANOva. Aquí se configuran tanto los efectos fijos y aleatorios de la prueba (tabla 3). 
Tabla 3. ANOVA prueba experimental

\begin{tabular}{l|c|c|c|c|c}
\multicolumn{1}{c|}{ Fuente } & GL & SC & MC & F & P \\
\hline Técnica & 3 & 31,844 & 10,615 & 0,47 & 0,710 \\
\hline Dominancia & 3 & 90,344 & 30,115 & 1,33 & 0,323 \\
\hline Técnica*Dominancia & 9 & 203,031 & 22,559 & 3,63 & 0,012 \\
\hline Error & 16 & 99,500 & 6,219 & & \\
\hline Total & 31 & 424,719 & & & \\
\hline & $\mathrm{r}$ & & \\
\hline
\end{tabular}

Fuente: Elaboración propia.

\section{INTERPRETACIÓN Y DISCU- SIÓN DE LOS RESULTADOS}

A partir del análisis de la ANOva (ver tabla 3), se evidencia un modelo cuyo ajuste es R-cuadrado $76,57 \%$ y R-cuadrado ajustado $54,61 \%$, y un MSE de 6,219.

Los niveles de ajuste alcanzados son aceptables para diseños de experimentos de este estilo que son considerados en el medio de la psicología experimental, en donde la variabilidad se asocia directamente con la conducta del ser humano. Sin embargo, resulta pertinente realizar nuevas corridas para verificar los resultados obtenidos y mejorar el nivel de ajuste.

$\mathrm{Al}$ verificar las tres pruebas de hipótesis planteadas en el numeral III y compararlas con el valor $p$ obtenido en el ANOva (ver tabla 3 ), se puede determinar lo siguiente:
El factor aleatorio: Técnica creativa. No contribuye significativamente a la variación de la calidad de las ideas generadas. Es importante resaltar que por haberse considerado la técnica como un factor aleatorio, esta conclusión tiene validez para las diferentes técnicas de generación de ideas existentes.

El factor fijo: Estilo cognitivo o dominancia cerebral. No contribuye significativamente a la calidad de las ideas generadas.

Interacción entre factor fijo y factor aleatorio: Es decir, la interacción entre la técnica y el estilo cognitivo dominante. Estos influyen significativamente sobre la calidad de las ideas generadas y esta información es soportada por los indicadores de ajuste del modelo.

A diferencia de los postulados propuestos por autores como Howard 
et al. (2010), Kohn, Paulus y Choi (2011) y Mak y Shu (2008), quienes afirman que las ideas se generan a través de la aplicación de técnicas creativas, elcasode estudio realizado evidenció que las técnicas creativas, desarrolladas según la propuesta de Vieira, Alves y Duboc (2012), consideradas como factor aleatorio del experimento, no inciden individualmente sobre la generación de ideas de calidad, siendo la calidad determinada según la propuesta de Reinig y Briggs (2013).

Contrastando los resultados obtenidos en el caso de estudio con los postulados de autores como Dane, Baer, Pratt y Oldham (2011), Liikkanen y Perttula (2006) y Nijstad, Stroebe y Lodewijkx (2002), quienes afirman que la generación de ideas en el ser humano se relaciona con los aspectos cognitivos propios de su condición, se encuentra que el estilo cognitivo - desarrollado según la propuesta de Herrmann (1991), el test de la Dra. Benziger y considerado en el caso de estudio como un factor fijo - no tiene incidencia particular o individual sobre la generación de ideas de calidad, determinada esta según la propuesta de Reining y Briggs (2013).

El caso de estudio evidenció que para esta empresa del sector gráfico de Medellín la generación de ideas se ve influenciada tanto por factores cognitivos de los colaboradores como por las técnicas creativas que se utilizan. Esto provee indicios sobre la importancia de considerar aspectos metodológicos, que estimulen al ser humano (técnicas creativas), y psicológicos, que determinan la forma en la que se procesa la información.

Con el objetivo de mejorar los niveles de ajuste del modelo, es pertinente considerar la inclusión de aspectos psicológicos adicionales del ser humano, entre los que se podría considerar la motivación y la personalidad, así como la aplicación de variables latentes para modelar factores psicológicos relacionados con la conducta del mismo, como puede ser para este caso de estudio el estilo cognitivo: un factor en donde el ser humano actúa como muestra y como juez.

\section{CONCLUSIONES \\ Y RECOMENDACIONES}

Tomando como referencia los estilos cognitivos, los seres humanos y la forma en la que ellos procesan la información, pueden ser clasificados a partir de teorías como la especialización hemisférica, planteada por Springer y Deutsch (1988), las inteligencias múltiples de Gardner y el modelo del cere- 
bro total propuesto por Herrmann (1991). Estas permiten caracterizar al individuo y su comportamiento en la gestión del conocimiento y la generación de ideas.

Los juegos gerenciales, una técnica propuesta por Gómez (2010) como herramienta de gestión del conocimiento, permiten la simulación de ambientes experimentales con limitaciones (debido a la complejidad de la realidad), que pueden ser modelados cuantitativamente mediante herramientas de diseño de experimentos. Esto facilita darle validez estadística al proceso, para adaptarlo al ambiente organizacional.

La calidad de las ideas generadas por el ser humano tiene incidencia directa sobre la innovación y su etapa preliminar, tal como se planteó en la revisión de literatura realizada para esta investigación. En este trabajo, que se ha enfocado en una empresa del sector gráfico de Medellín, se detectó que la calidad de las ideas, dependiendo de la etapa en el proceso de innovación en el que se encuentre la organización y la naturaleza del problema a resolver, tiene diferentes alcances y métodos de medición. Entre ellos se encuentra el método propuesto por Reinig y Briggs (2013) o el de Shah, Smith y Vargas (2003).
En esta investigación se concluye que la generación de ideas de calidad está determinada tanto por las técnicas creativas implementadas, como por el estilo cognitivo del individuo. Este es un aspecto importante para la administración de los recursos humanos, debido a que, teniendo en cuenta este criterio, es posible llegar a influenciar de manera efectiva la actividad de generación de ideas. Esto se puede hacer mediante la conformación de los equipos y la selección de los métodos que mejor se adapten a la naturaleza y al estilo cognitivo de sus integrantes. Esta es una labor que puede permitir así una mejor gestión de la innovación.

\section{REFERENCIAS}

Alles, M. A. (2005). Gestión por competencias: el diccionario. Buenos Aires: Granica.

Ashby, F. G., Isen, A. M., \& Turken, A. U. (1999). A neuropsychological theory of positive affect and its influence on cognition. Psychological Review, 106(3), 529-550.

Bueno, A. del M. (2007). Gestión del conocimiento. Madrid: Thomson-Paraninfo.

Carrión, J. L. (1995). Manual de neuropsicología humana. Madrid: Siglo XXI.

Chernigovskaya, T. V., Gavrilova, T. A., Voinov, A. V., \& Strelnikov, 
K. N. (2005). Sensorimotor and cognitive laterality profiles. $\mathrm{Hu}$ man Physiology, 31(2), 142-149. Comisión Europea (1995). Libro verde de la innovación. Bruselas: Oficina de Publicaciones Oficiales de las Comunidades Europeas.

Cooper, R. G. (1990). Stage-gate systems: a new tool for managing new products. Business $\mathrm{Ho}-$ rizons, 33(3), 44-54.

Dane, E., Baer, M., Pratt, M. G., \& Oldham, G. R. (2011). Rational versus intuitive problem solving: How thinking "off the beaten path" can stimulate creativity. Psychology of Aesthetics, Creativity, and the Arts, 5(1), 3-12.

De Brentani, U., \& Kleinschmidt, E. J. (2004). Corporate culture and commitment: impact on performance of international new product development programs. Journal of Product Innovation Management, 21(5), 309-333.

De Zubiría Samper, J., Cortés, J. S. P., \& Rodríguez, M. P. (2007). Los estilos cognitivos en el Instituto Alberto Merani. Recuperado de http://www.institutomerani.edu. co/publicaciones/tesis/Los $\% 20$ Estilos\%20Cognitivos\%20 en\%20el\%20IAM.pdf

Formichella, M. (2005). La evolución del concepto de innovación y su relación con el desarrollo. Argentina: INTA. Recuperado de http://www.upc.gob.bo/upc/ biblioteca/docs/innovacion/evolucion $\% 20$ concepto $\% 20 \mathrm{de} \% 20$ innovacion.pdf

Gardié, O. (1998). Cerebro total y visión holístico-creativa de la educación. Estudios Pedagógicos (Valdivia), 24, 79-87.

Gardner, H. (1994). Estructuras de la mente: la teoría de las inteligencias múltiples. México: Fondo de Cultura Económica.

Gómez, A., M. C. (2009). De afuera hacia adentro. HSM Management, 4, 42-47.

Gómez Álvarez, M. C. (2010). Definición de un método para el diseño de juegos orientados al desarrollo de habilidades gerenciales como estrategia de entrenamiento empresarial. Tesis de maestría en Ingeniería Administrativa. Recuperado de http://www.bdigital.unal.edu. co/1968/1/32242923.20101.pdf

Hagerstrand, T. (1968). The diffusion of innovations. International Encyclopedia of Social Sciences, 4, 174-178.

Herrmann, N. (1991). The creative brain. The Journal of Creative Behavior, 25(4), 275-295.

Howard, T. J., Dekoninck, E. A., \& Culley, S. J. (2010). The use of creative stimuli at early stages of industrial product innovation. Research in Engineering Design, 21(4), 263-274. 
Jardón, C. M. (2011). Innovación empresarial y territorio: una aplicación a Vigo y su área de influencia. EURE (Santiago), 37(112), 115-139.

Johannessen, J.-A., Olsen, B., \& Olaisen, J. (1999). Aspects of innovation theory based on knowledge-management. International Journal of Information Management, 19(2), 121-139.

Kohn, N. W., Paulus, P. B., \& Choi, Y. (2011). Building on the ideas of others: An examination of the idea combination process. Journal of Experimental Social Psychology, 47(3), 554-561.

Liikkanen, L. A., \& Perttula, M. K. (2006). Contextual cueing and verbal stimuli in design idea generation. En J. S. Gero (Ed.). Design Computing and Cognition (pp. 619-631). Netherlands: Springer.

Lin, T. C., \& Huang, C. C. (2008). Understanding knowledge management system usage antecedents: An integration of social cognitive theory and task technology fit. Information \& Management, 45(6), 410-417.

MacLean, P. D. (1990). The triune brain in evolution: Role in paleocerebral functions. Nueva York: Springer.

Mak, T. W., \& Shu, L. H. (2008). Using descriptions of biological phenomena for idea generation.
Research in Engineering Design, 19(1), 21-28.

Melo Martínez, O. O., López Pérez, L. A., \& Melo Martínez, S. E. (2007). Diseño de experimentos: métodos y aplicaciones. Bogotá: Universidad Nacional de Colombia, Facultad de Ciencias.

Méndez, R. (2002). Innovación y desarrollo territorial: algunos debates teóricos recientes. EURE (Santiago), 28(84), 63-83.

Montgomery, D. C. (2005). Diseño $y$ análisis de experimentos. México: Limusa.

Nijstad, B. A., Stroebe, W., \& Lodewijkx, H. F. (2002). Cognitive stimulation and interference in groups: Exposure effects in an idea generation task. Journal of Experimental Social Psychology, 38(6), 535-544.

Nonaka, I., \& Takeuchi, H. (2000). La empresa creadora de conocimiento. Gestión del Conocimiento. Bilbao: Harvard Deusto Bussines Review, 23-50.

OECD (2005). Manual de Oslo, $3^{\mathrm{a}} \mathrm{ed}$. Luxembourg: OECD Publishing.

Pérez, B., \& Carrillo, E. (2000). Desarrollo local: manual de uso. Madrid: ESIC-Federación Andaluza de Municipios y Provincias.

Porter, M. E. (1985). Competitive advantage. Creative and sustaining. Nueva York: The Free Press. 
Prahalad, C. K., \& Hamel, G. (1990). The core competence of the corporation. Harvard Business Review, 68(3), 79-91.

Reinig, B. A., \& Briggs, R. O. (2013). Putting quality first in ideation research. Group Decision and Negotiation, 22(5), 943-973.

Ricardo, D., Gonner, E. C. K., \& Li, Q. (1817). On the principles of political economy and taxation. Londres: Black Horse Court.

Riding, R. J., Glass, A., \& Douglas, G. (1993). Individual differences in thinking: Cognitive and neurophysiological perspectives. Educational Psychology, 13(3-4), 267-279.

Riesco González, M. (2006). El negocio es el conocimiento. España: Díaz de Santos.

Schumpeter, J. A. (1934). The theory of economic development: An inquiry into profits, capital, credit, interest, and the business cycle. University of Illinois at Urbana-Champaign's academy for entrepreneurial leadership historical research reference in entrepreneurship. Recuperado de http://papers.ssrn. com/sol3/papers.cfm?abstract id $=1496199 \&$

Sperry, R. W. (1968). Hemisphere deconnection and unity in conscious awareness. American Psychologist, 23(10), 723-733.
Springer, S. P., \& Deutsch, G. (1988). Cerebro izquierdo, cerebro derecho. Madrid: Alianza.

Tennant, M. (2006). Psychology and adult learning. Nueva York: Routledge.

Verhees, F. J., \& Meulenberg, M. T. (2004). Market orientation, innovativeness, product innovation, and performance in small firms. Journal of Small Business Management, 42(2), 134-154.

Vieira, E. R., Alves, C., \& Duboc, L. (2012). Creativity Patterns Guide: Support for the Application of Creativity Techniques in Requirements Engineering. En M. Winckler, P. Forbrig, \& R. Bernhaupt (Eds.). HumanCentered Software Engineering (vol. 7623, pp. 283-290). Berlín, Heidelberg: Springer Berlin Heidelberg.

Villaseñor, J. A. (2011). Pruebas de bondad de ajuste para distribuciones con parámetro de forma. Recuperado de http://amestad. mx/pdf/conferencias/2011/Jose Villasenor_Alva_Sept_2011.pdf Wilson, J. O., Rosen, D., Nelson, B. A., \& Yen, J. (2010). The effects of biological examples in idea generation. Design Studies, 31(2), 169-186.

Yin, R. K. (1992). The case study method as a tool for doing evaluation. Current Sociology, 40(1), 121-137. 